\title{
Cryotherapy and Topical Minocycline as Adjunctive Measures to Control Pain After Third Molar Surgery: An Exploratory Study
}

\author{
Savannah Gelesko, DDS ${ }^{*}$, Leann Long, MS ${ }^{\dagger}$, Jan Faulk, DDS ${ }^{\ddagger}$, Ceib Phillips, PhD, MPH ${ }^{\S}$, \\ Carolyn Dicus, DMD, MD\|, and Raymond P. White Jr, DDS, PhD" \\ ${ }^{\dagger}$ PhD Candidate, Department of Biostatistics, Gillings School of Global Public Health, University \\ of North Carolina, Chapel Hill, NC \\ ¥Clinical Associate Professor, Department of Oral and Maxillofacial Surgery, School of Dentistry, \\ University of North Carolina, Chapel Hill, NC \\ §Professor, Department of Orthodontics, School of Dentistry, University of North Carolina, Chapel \\ Hill, NC \\ "Resident, Department of Oral and Maxillofacial Surgery, School of Dentistry, University of North \\ Carolina, Chapel Hill, NC \\ IDalton L. McMichael Distinguished Professor, Department of Oral and Maxillofacial Surgery, \\ School of Dentistry, University of North Carolina, Chapel Hill, NC
}

\begin{abstract}
Purpose-To assess the impact of cryotherapy or topical minocycline on patients' perceptions of recovery from pain after third molar surgery in an exploratory comparative-effectiveness study.

Patients and Methods-Subjects aged at least 14 years who were having all 4 third molars removed were enrolled in 3 separate institutional review board-approved studies. Study groups included subjects treated with a passively applied cold wrap for 24 hours postoperatively, subjects treated with topical minocycline during surgery, and subjects enrolled in a nonconcurrent comparison group who had received neither topical minocycline nor directed cryotherapy. Third molar surgery was performed in all cases by trained surgeons using the same protocol. An exact Kruskal-Wallis test was used to compare the distributions of the worst and average pain scores and a Fisher exact test to compare verbal responses from Gracely pain scales among the 3 groups for postsurgical days (PSDs) 1 to 3 .
\end{abstract}

Results-This study comprised 51 cryotherapy subjects (2005-2009), 63 minocycline subjects (2003-2004), and 92 comparison-group subjects (2002-2006) who were treated at academic centers and in community practices across the United States $(\mathrm{N}=206)$. Demographic descriptors were similar among all groups. For PSDs 1 through 3 (unadjusted), the highest scores for worst pain (6-7 [out of 7] on Likert-type scale) were reported less frequently in each of the study groups than in subjects in the comparison group, although the numbers of subjects reporting the highest scores were few. The distribution of pain outcomes was significantly different among the 3 groups for worst pain and affective words on PSD 1 ( $P=.04$ for both). However, the small number of

\footnotetext{
(C) 2011 American Association of Oral and Maxillofacial Surgeons

Address correspondence and reprint requests to Dr White: Department of Oral and Maxillofacial Surgery, School of Dentistry, University of North Carolina, Chapel Hill, NC 27599; ray_white@dentistry.unc.edu.

${ }^{*}$ Currently, Resident, Department of Oral and Maxillofacial Surgery, School of Dentistry, Oregon Health and Science University, Portland, OR; Formerly, Dental Student, University of North Carolina, School of Dentistry, University of North Carolina, Chapel Hill, NC.
} 
subjects who reported the highest pain scores precluded adequate multivariate statistical analyses for all outcomes on PSD 1 to 3.

Conclusions-Data from this exploratory study suggest that adjunctive therapy to decrease postoperative pain — cryotherapy or topical minocycline-might be effective at moderating the patient's highest pain levels after third molar surgery. The topic should be studied further in a multicenter, prospective, randomized trial.

Most patients have pain, swelling, and dysfunction related to inflammation for a short, predictable period after third molar surgery. ${ }^{1,2}$ Because of the consistency of these outcomes, recovery after third molar surgery is often used as a model for studying the efficacy of analgesic and anti-inflammatory drugs for this and other procedures affecting bone and connective tissue. This model is recommended by the US Food and Drug Administration for these reasons and because patients having third molar surgery are usually healthy without complicating medical conditions. ${ }^{3}$

Almost all patients report taking medications for pain, usually an opioid in combination with a nonsteroidal anti-inflammatory drug (NSAID), for at least the first few days after third molar surgery. ${ }^{4}$ In addition to prescribing these medications for control of pain, surgeons and other clinicians seek adjunctive measures to reduce inflammation and its related pain postoperatively. Cryotherapy is used routinely in sports medicine for its beneficial effects, including a reduction in levels of inflammation and pain. ${ }^{5,6}$ In addition to their antimicrobial properties, the second-generation, chemically modified tetracyclines doxycycline and minocycline have been shown to reduce pain and inflammation after surgery. ${ }^{7,8}$ Topical formulations of minocycline, incorporated into a bioresorbable polymer allowing release of the drug over time, have been effective in reducing inflammation associated with periodontal inflammatory disease. ${ }^{9}$ If cryotherapy or topical minocycline can reduce inflammation and its sequelae after third molar surgery, recovery from postoperative pain might be improved, possibly decreasing the need for pain medications for a protracted period after surgery.

The perceptions of recovery from pain of subjects treated at academic clinical centers and in community practices with cryotherapy or topical minocycline were compared with the responses from a comparison group of subjects with no specific instructions for cryotherapy and no topical minocycline at surgery.

\section{Patients and Methods}

Healthy subjects (American Society of Anesthesiologists status I or II), aged 14 to 40 years, scheduled for removal of 4 third molars were recruited and voluntarily enrolled in institutional review board-approved prospective clinical studies taking place at multiple academic clinical centers and in community practices across the United States. The clinical study sites were selected based on the clinicians' prior experience with similar study protocols and willingness to participate. The intent of this analysis is to compare the effectiveness of 3 separate clinical protocols with respect to patient recovery from pain.

\section{CRYOTHERAPY STUDY}

Subjects were treated at 1 academic center between 2005 and 2009. Subjects were given a passively applied thermal wrap and instructed to wear the device continuously for at least 24 hours (either Patient's PAL [Rogers, AR; product no longer available] or Cool Jaw [Medico International, Palmer, PA]). 


\section{TOPICAL MINOCYCLINE STUDY}

Subjects were treated at 2 academic clinical centers and in 2 community practices between 2003 and 2004. Patients reporting current tobacco use or antibiotic use within the last month were excluded from enrollment in the topical minocycline study. For subjects in the topical minocycline study, $1 \mathrm{mg}$ of topical minocycline was delivered through sustained-release microspheres to each mandibular surgery site after third molar removal (Arestin; OraPharma, Warminster, PA). Stavropoulos et $\mathrm{al}^{10}$ have provided additional details on the minocycline study.

\section{COMPARISON STUDY}

A comparison nonconcurrent group of subjects were treated at 3 academic centers and in 2 community practices between 2002 and 2006, a time frame close to that of the 2 study groups. The passive cryotherapy devices were not available for the subjects in the comparison group, although other cryotherapy measures such as a chemically activated cold pack that required active patient participation may have been used. The topical minocycline drug was not available for use in the subjects in the comparison group. Subjects were not randomized to the treatments (cryotherapy or topical minocycline) or to the comparison group.

Third molar surgery for all subjects was conducted with the same protocol and included procedures commonly performed in the United States, such as intravenous anesthesia, and rotary instrumentation for bone removal for buccal access to mandibular third molars, as reported by White et al. ${ }^{1}$ In academic centers surgery was carried out by residents with at least 1 year of dentoalveolar experience after dental school or by full-time faculty, specialty board-certified surgeons who were Fellows of the American Association of Oral and Maxillofacial Surgeons. Third molar surgery in community practices was performed by specialty board-certified surgeons, all Fellows of the American Association of Oral and Maxillofacial Surgeons.

In all 3 groups, preoperative data were collected on all subjects including demographics (age, gender, ethnicity). Data recorded immediately postoperatively addressed the extensiveness of the surgery: the total surgery time in minutes, bone removal from both mandibular third molars or not, the anatomic relationship of lower third molars either at or below the occlusal plane, and the surgeon's estimate of the degree of difficulty for each third molar rated on a scale from 1 to 7 for a possible score ranging from 4 to $28 .{ }^{1,2}$

After third molar removal, subjects in all 3 groups were given a condition-specific instrument in the form of a diary, to be completed once each postsurgical day (PSD) for 14 days to assess their perception of recovery from pain. ${ }^{11,12}$ The subjects' levels of worst pain and average pain over each postoperative 24-hour period were assessed on a 7-point Likerttype scale anchored with verbal descriptive anchors "no pain" and "worst pain imaginable." The sensory intensity of pain and the unpleasantness of pain were measured by use of Gracely scales with 13 verbal descriptors. ${ }^{1}$ Pain intensity was anchored by "nothing" and "extremely intense," and pain unpleasantness was anchored by "neutral" and "very intolerable." The report of White et al ${ }^{1}$ provides additional details on data collection and management, which were the same for all groups.

For the Likert-type scales, pain scores of 6 to 7 (out of 7) were categorized as severe pain, 3 to 5 as moderate pain, and 1 to 2 as little pain. For the Gracely scales, the affective words "very distressing," "intolerable," and "very intolerable" were categorized as "clinically undesirable." The affective words "slightly distressing," "very unpleasant," "distressing," "very annoying," and "slightly intolerable" were categorized as "clinically acceptable." The affective words "neutral," "slightly unpleasant," "slightly annoying," "unpleasant," and 
"annoying" were considered "clinically desirable." For the Gracely scales, the sensory words "intense," "very intense," and "extremely intense" were categorized as "clinically undesirable." The sensory words "mild," "moderate," "barely strong," "slightly intense," and "strong" were categorized as "clinically acceptable." The sensory words "nothing," "faint," "very weak," "weak," and "very mild" were considered "clinically desirable."

We used $\chi^{2}$ and Cochran-Mantel-Haenszel row mean score statistics to compare the demographics (gender, ethnicity, and age) and the extensiveness of the surgery (bone removal from both lower third molars, mandibular occlusal plane position, total surgery time, and surgeon's assessment of total difficulty) among the 3 groups.

The primary outcome analyses of worst and average pain scores and affective and sensory word categorizations were focused on the first 3 PSDs, the time frame when clinicians would expect pain outcomes to be most affected by the adjunctive measures (ie, cryotherapy or topical minocycline). Among the 3 groups, the Fisher exact test was used to compare the proportion of word categorizations, and an exact Kruskal-Wallis test was used to compare the distribution of the pain outcomes from the Likert-type scales. The level of significance was set at .05 for all analyses.

\section{Results}

During the 7 years from 2002 to 2009, 206 subjects reported on how having surgery for removal of all 4 third molars affected their quality of life, including outcomes for postoperative pain. The cryotherapy group consisted of 51 subjects treated at 1 academic center from 2005 to 2009; the topical minocycline group consisted of 63 subjects treated at 2 academic centers and in 2 community practices from 2003 to 2004; the comparison group consisted of 92 subjects treated at 3 academic centers and in 2 community practices from 2002 to 2006.

The median age at surgery was similar for the 3 groups (20 or 21 years) (Table 1). More women had surgery than men, $57 \%$ versus $43 \%$, and white subjects predominated as compared with subjects of other races, $80 \%$ versus $20 \%$. There were no statistically significant demographic differences among the 3 groups (Table 1). The median total surgery time was significantly different among the groups $(P=.03)$ and varied from 25 to 27 minutes. The surgeon's assessment of the degree of difficulty of the surgery ranged from 12 to 14 , on average, among the groups. At least two-thirds of subjects in all groups had bone removal for both mandibular third molars, with the highest frequency being reported for the comparison group, at $83 \%$. Significantly more subjects in the cryotherapy group had all third molars at the occlusal plane (62\%) as compared with those in the topical minocycline group (37\%) and comparison group $(52 \%)(P=.04)$.

The frequencies of subjects taking pain medications differed little between study groups. Almost all subjects, at least $96 \%$ in each group, reported using pain medications (opioid, NSAID, or both) on PSD 1 (data not shown). By PSD 3, these percentages fell, but at least $83 \%$ of subjects in each group reported taking pain medication.

The distribution of responses for the comparison subjects was shifted toward the highest scores for worst pain and "clinically undesirable" affective and sensory words on PSD 1 to 3 (Fig 1; Tables 2,3). The distribution of pain outcomes was significantly different among the 3 groups for worst pain and affective words on PSD 1 ( $P=.04$ for both). The distribution of responses for the cryotherapy and minocycline subjects was shifted toward the lowest scores for average pain on PSD 1 to 3. 
Fewer subjects treated with directed cryotherapy or topical minocycline reported scores for worst pain of 6 to 7 (out of 7) as compared with subjects in the comparison group (Fig 1). For example, on PSD 1, only $8 \%$ of cryotherapy and $19 \%$ of topical minocycline subjects reported scores of 6 to 7 for worst pain in the past 24 hours, compared with $28 \%$ of subjects in the comparison group. The beneficial impact of cryotherapy and topical minocycline treatment on worst pain persisted through PSD 3 (Fig 1). For example, on PSD 3, 7\% of the cryotherapy subjects and $8 \%$ of the topical minocycline subjects reported scores of 6 to 7 for worst pain in the past 24 hours, whereas $19 \%$ of the subjects in the comparison group reported these highest levels of pain. A similar distributional shift in the pattern of reported responses away from the highest scores persisted for average pain. For example, on PSD 1, $35 \%$ of cryotherapy subjects and $30 \%$ of topical minocycline subjects reported their average pain being at the lowest pain levels, 1 to 2 of 7, compared with $16 \%$ of subjects in the comparison group (Fig 2).

The Gracely scales' clinically undesirable affective words and sensory words were also reported less frequently on PSD 1 to 3 in the cryotherapy and topical minocycline subjects than in the comparison subjects (Tables 2, 3). For example, on PSD 1, affective words considered clinically undesirable were reported by $0 \%$ of the cryotherapy subjects and $2 \%$ of the topical minocycline subjects, as compared with $11 \%$ of the comparison subjects. On PSD 3, clinically undesirable affective words were reported by $4 \%$ of the cryotherapy subjects and 5\% of the topical minocycline subjects, as compared with $11 \%$ of the comparison subjects.

\section{Discussion}

Findings from this exploratory comparative-effectiveness study of 3 study protocols analyzing postoperative data from 206 healthy, young adult subjects, treated at academic clinical centers and in community practices across the United States, suggested that topical minocycline placed in third molar extraction sites during surgery or passively applied cryotherapy postoperatively may be effective as adjunctive measures added to prescribed analgesic medications to control postoperative pain. These adjunctive measures to pain medication resulted in a distributional shift in pain scores, away from the most severe pain levels as reported by subjects in diaries completed for PSD 1 to 3 .

The distribution of pain scores for PSD 1 to 3 reported by the 92 subjects in the comparison group was similar to distributions reported by White et $\mathrm{al}^{1}$ and Phillips et $\mathrm{al}^{2}$ for 545 subjects studied between 1997 and 2001. Although the pain scores reported by the comparison group were shifted toward more severe pain when compared with the cryotherapy and topical minocycline groups, subjects in the treatment groups designed to moderate postoperative pain were not randomized to a treatment group (cryotherapy or topical minocycline) or the alternative of no adjunctive intervention. In addition, the small number of subjects who reported the highest pain scores on the Likert or Gracely scales precluded adequate multivariate statistical analyses for the pain outcomes, and the comparison among the 3 groups did not include adjustment for potential covariates that differed among the 3 groups that could affect a subject's pain response. For example, subjects in the comparison group had a greater frequency of bone removal, suggesting more difficult surgery as compared with treatment subjects. However, subjects in the minocycline group had significantly more mandibular third molars below the occlusal plane, a longer surgery time, and a higher surgeon's estimate of degree of difficulty, suggesting more difficult surgery for these subjects as compared with the other study groups. Because the design of our study has these limitations, the impact of cryotherapy or topical minocycline on postoperative pain should be studied further in a multisite, concurrent clinical trial with subjects randomized to cryotherapy, topical minocycline, both interventions, or neither. 


\section{IS THERE A BIOLOGICAL BASIS FOR CRYOTHERAPY MODERATING POSTOPERATIVE PAIN?}

Cryotherapy is the therapeutic application of cold to remove heat from the body. ${ }^{13}$ The depth of the target tissue for cryotherapy is important to consider when deciding on mode of application. Skin and superficial tissues cool quickly, whereas cold penetration in deeper tissues is slower and less intense. In their study of temperature change at the quadriceps muscle, Enwemeka et $\mathrm{al}^{6}$ found significant falls in temperature of the skin and tissues $1 \mathrm{~cm}$ deep after just 8 minutes of cold pack application. The deeper tissues cooled only during warming of the more superficial tissues, and there was no significant cooling of deeper tissues $(2-3 \mathrm{~cm})$ during treatment. Possoff ${ }^{14}$ reported human cheek thickness as a mean of $15 \mathrm{~mm}$ and a range of 10 to $19 \mathrm{~mm}$. He found only a $1^{\circ} \mathrm{C}$ decrease in alveolar mucosa temperature after 30 minutes of cryotherapy (ice bag wrapped in towel). Anatomic variation in subjects' jaw shape could impact the effectiveness of any cryotherapy.

\section{HOW DOES CRYOTHERAPY DECREASE A PATIENT'S PAIN EXPERIENCE AFTER SURGERY?}

Cryotherapy raises the threshold for pain fibers and reduces nerve conduction velocity. Superficial nerves are impacted most often, and sensory fibers are blocked before motor fibers. Even after removal of the cryotherapy, the effect may last up to 30 minutes. ${ }^{15}$ The literature is unclear on the duration of cryotherapy necessary for clinical improvement; 9- to 15-minute intervals have been reported as successful for pain reduction, but at least 12 minutes is recommended to achieve local numbness. ${ }^{16}$ Changes in conduction of sensation occur at higher temperatures than the impact of cold in moderating inflammation.

Incorrect application of cryotherapy can result in damage to the skin, namely frostbite. A barrier layer of material between the cold substance and the skin minimizes this rare complication. Freezing and reapplication of single-use chemical cold packs should be avoided because the toxic refrigerant may leak, resulting in tissue damage. ${ }^{17,18}$ Patients with peripheral vascular disease such as Raynaud disease or type 1 diabetes mellitus should use cryotherapy with caution even for the relatively short postoperative interval.

Cryotherapy has been extensively studied and recommended for many clinical applications including soft tissue trauma after injury and after surgery, as well as for orthopedic rehabilitation. ${ }^{19}$ Multiple modes of cryotherapy have been described (crushed ice, an ice pack, frozen peas, a chemical gel pack, and the application in this study, a gel pack that must be frozen before application). The literature is inconclusive as to which form of cryotherapy is the most ideal treatment; however, all seem to produce temperature reduction adequate for analgesia. In their critical literature review examining 22 randomized controlled clinical trials including multiple modes of cryotherapy for patients after injury and after surgery, Hubbard and Denegar ${ }^{20}$ concluded that cryotherapy is effective in reducing pain. However, several of the studies they reviewed were confounded by concomitant use of compression, requiring —at a minimum - the patient's active participation in the treatment. ${ }^{20,21}$

The literature review on cryotherapy after intraoral surgical procedures by Greenstein ${ }^{16}$ was inconclusive with respect to the clinical benefits of cryotherapy including pain outcomes. The oral and maxillofacial surgery literature contains minimal data regarding the effectiveness of cryotherapy postoperatively for intraoral procedures, and conflicting evidence exists on whether cryotherapy reduces postoperative pain. ${ }^{22-25}$

Our subjects were instructed to use their passively applied thermal wraps continuously for 24 hours or through the morning the day after surgery. The thermal wraps took approximately 40 minutes to freeze initially and then stayed cold for about 20 to 25 minutes. The application, then, was intermittent because the thermal wrap had to be refrozen before 
reapplication. However, once applied, the device was passive; a subject's hands were free, a distinct difference from methods of cryotherapy requiring a patient to actively hold the device in place, potentially reducing compliance with treatment including the total time the cryotherapy was applied.

\section{IS THERE A BIOLOGICAL BASIS FOR TOPICAL MINOCYCLINE IN PAIN REDUCTION?}

In addition to the bacteriostatic properties of tetracyclines, the chemically modified tetracycline analogs have multiple non-antibiotic properties including inhibition of lymphocyte proliferation, inhibition of proinflammatory mediators, and stimulation of bone formation. ${ }^{7}$ The tetracycline carboxamide ring system contains upper and lower peripheral zones that may be modified to affect both antimicrobial and nonantibiotic properties. Minocycline, like doxycycline, is a chemically modified tetracycline analog, created by modification of the upper peripheral zone, affecting the biological properties but not the antimicrobial properties. ${ }^{7}$ The work of Bastos et $\mathrm{al}^{8}$ using rat models helped to characterize the specific antinociceptive and anti-inflammatory effects of minocycline and doxycyecline. Minocycline inhibits the nociception phase associated with inflammatory mediator synthesis; thus they propose the related mechanism to be inhibition of synthesis of these inflammatory mediators. ${ }^{8}$

For over a decade, chemically modified tetracycline analogs have been used at subantimicrobial dosages for their host modulation properties such as indirect inhibition of matrix metalloproteinases. ${ }^{26}$ The seminal experiments of Golub et $\mathrm{al}^{27,28}$ in germ-free diabetic rat models showed that tetracyclines have clear anticollagenase properties, independent of their antimicrobial activities. Thus minocycline has been used extensively to treat chronic inflammatory diseases including periodontal disease, inflammatory acne, and mild rheumatoid arthritis. ${ }^{8,9}$ In this study the formulation and dosage of the topical minocycline made the drug available at the surgical site for the 3-day postoperative study time frame.

This study has multiple limitations. Although topical minocycline in an extended-release vehicle was not available for use in subjects in the comparison group, surgeons treating subjects in the comparison group may have used a form of cryotherapy other than a passively applied thermal wrap, and these applications were not recorded. Subjects were young, healthy adults, treated at multiple academic clinical centers and in community practices across the country, a reasonable representation of the majority of patients undergoing third molar removal with contemporary surgical techniques. However, study subjects were not representative of the US population, nor were subjects studied concurrently. The data may not be applicable to older patients having third molar surgery. All surgery was performed by trained oral and maxillofacial surgeons with techniques commonly used in the United States. Outcomes may differ if surgery was performed by clinicians without extensive surgical training. The study did not control for clinicians' use of corticosteroids in patients at surgery; corticosteroids have been shown to decrease postoperative pain. ${ }^{29,30}$ Although none of the cryotherapy subjects received corticosteroids, data on corticosteroid use were not available for the minocycline subjects. Most subjects used pain medications for PSD 1 to 3, usually an opioid or an NSAID, but total dosages among the study groups were not recorded. ${ }^{4}$ Outcomes were not adjusted for tobacco use; tobacco use postoperatively has been shown to increase postoperative pain. ${ }^{31}$ Despite the limitations, the data do suggest a possible beneficial impact of cryotherapy or topical minocycline, moderating postoperative pain in the first few days after third molar surgery. The topic should be studied further in a multicenter, prospective, randomized trial. 


\section{Acknowledgments}

The Oral and Maxillofacial Surgery Foundation, American Association of Oral and Maxillofacial Surgeons, and Dental Foundation of North Carolina provided funding for these analyses.

The authors offer special thanks to the surgeons and their patients who volunteered to provide data for this analysis. They thank Ms Debora Price for assistance in managing data for this project.

\section{References}

1. White RP Jr, Shugars DA, Shafer DM, et al. Recovery after third molar surgery: Clinical and health related quality of life outcomes. J Oral Maxillofac Surg. 2003; 61:535. [PubMed: 12730831]

2. Phillips C, White RP Jr, Shugars D, et al. Risk factors associated with prolonged recovery and delayed clinical healing after third molar surgery. J Oral Maxillofac Surg. 2003; 61:1436. [PubMed: 14663809]

3. Averbach M, Katzper M. A search for sex differences in response to analgesia. Arch Intern Med. 2000; 160:3424. [PubMed: 11112235]

4. Snyder M, Shugars DA, White RP Jr, et al. The role of pain medication after third molar surgery on recovery for lifestyle and oral function. J Oral Maxillofac Surg. 2005; 63:1130. [PubMed: 16094580]

5. MacAuley DC. Ice therapy: How good is the evidence? Int J Sports Med. 2001; 22:379. [PubMed: 11510876]

6. Enwemeka CS, Allen C, Avila P, et al. Soft tissue thermodynamics before, during, and after cold pack therapy. Med Sci Sports Exerc. 2002; 34:45. [PubMed: 11782646]

7. Sapadin AN, Fleishmajer R. Tetracyclines: Nonantibiotic properties and their clinical implications. J Am Acad Dermatol. 2006; 54:258. [PubMed: 16443056]

8. Bastos LF, Merlo LA, Rocha LT, Coelho MM. Characterization of the antinociceptive and antiinflammatory activities of doxycycline and minocycline in different experimental models. Eur $\mathbf{J}$ Pharmacol. 2007; 576:171. [PubMed: 17719028]

9. Williams RC, Paquette DW, Offenbacher S, et al. Treatment of periodontitis by local administration of minocycline microspheres: A controlled trial. J Periodontol. 2001; 72:1535. [PubMed: 11759865]

10. Stavropoulos MF, Shugars DA, Phillips C, et al. The impact of topical minocycline with third molar surgery on clinical recovery and health related quality of life outcomes. J Oral Maxillofac Surg. 2006; 64:1059. [PubMed: 16781338]

11. Conrad SM, Blakey GH, Shugars DA, et al. Patients' perception of recovery after third molar surgery. J Oral Maxillofac Surg. 1999; 57:1288. [PubMed: 10555792]

12. Shugars DA, Benson K, White RP Jr, et al. Developing a measure of patient perceptions of shortterm outcomes of third molar surgery. J Oral Maxillofac Surg. 1996; 54:1402. [PubMed: 8957118]

13. Nadler SF, Weingand K, Kruse RJ. The physiologic basis and clinical applications of cryotherapy and thermotherapy for the pain practitioner. Pain Physician. 2004; 7:395. [PubMed: 16858479]

14. Possoff A. External thermal applications in postextraction therapy. J Am Dent Assoc. 1955; 50:157.

15. Lee JM, Warren MP, Mason SM. Effects of ice on nerve conduction velocity. Physiotherapy. 1978; 64:2. [PubMed: 628689]

16. Greenstein G. Therapeutic efficacy of cold therapy after intraoral surgical procedures: A literature review. J Periodontol. 2007; 78:790. [PubMed: 17470011]

17. Kujawska A, Kwaselow A, Pansare M. Unusual complication with use of an instant cold pack. Ann Allergy Asthma Immunol. 2009; 103:356. [PubMed: 19852203]

18. Stevens DM, D’Angelo JV. Frostbite due to improper use of frozen gel pack. N Engl J Med. 1978; 299:1415. [PubMed: 714122]

19. Bleakley C, McDonough S, MacAuley D. The use of ice in the treatment of acute soft-tissue injury: A systematic review of randomized controlled trials. Am J Sports Med. 2004; 32:251. [PubMed: 14754753] 
20. Hubbard TJ, Denegar CR. Does cryotherapy improve outcomes with soft tissue injury? J Athl Train. 2004; 39:278. [PubMed: 15496998]

21. Forouzanfar T, Sabelis A, Ausems S, et al. Effect of ice compression on pain after mandibular third molar surgery: A singleblind, randomized controlled trial. Int J Oral Maxillofac Surg. 2008; 37:824. [PubMed: 18603410]

22. Laureano-Filho JR, de Oliveira e Silva ED, Batista CL, et al. The influence of cryotherapy on reduction of swelling, pain, and trismus after third-molar extraction: A preliminary study. $\mathrm{J}$ Am Dent Assoc. 2005; 136:774. [PubMed: 16022042]

23. Bastian H, Soholm B, Marker P, et al. Comparative study of pain control by cryotherapy of exposed bone following extraction of wisdom teeth. J Oral Sci. 1998; 40:109. [PubMed: 9838745]

24. Forsgren H, Heimdahl A, Johansson B, et al. Effect of application of cold dressings on the postoperative course in oral surgery. Int J Oral Surg. 1985; 14:223. [PubMed: 3926665]

25. Van der Westhuijzen AJ, Becker PJ, Morkel J, et al. A randomized observer blind comparison of bilateral facial ice pack therapy with no ice therapy following third molar surgery. Int J Oral Maxillofac Surg. 2005; 34:281. [PubMed: 15741037]

26. Golub LM, Lee HM, Ryan ME, et al. Tetracyclines inhibit connective tissue breakdown by multiple non-antimicrobial mechanisms. Adv Dent Res. 1998; 12:12. [PubMed: 9972117]

27. Golub LM, Lee HM, Lehrer G, et al. Minocycline reduces gingival collagenolytic activity during diabetes: Preliminary observations and a proposed new mechanism of action. J Periodont Res. 1983; 18:516. [PubMed: 6315909]

28. Golub LM, Ramamurthy NS, McNamara TF, et al. Tetracylines inhibit tissue collagenase activity: A new mechanism in the treatment of periodontal disease. J Periodont Res. 1984; 19:651. [PubMed: 6098638]

29. Markiewicz MR, Brady MF, Ding EL, et al. Corticosteroids reduce postoperative morbidity after third molar surgery: A systematic review and meta-analysis. J Oral Maxillofac Surg. 2008; 66:1881. [PubMed: 18718396]

30. Tiwana PS, Foy SP, Shugars DA, et al. The impact of intravenous corticosteroids with third molar surgery in patients at high risk for delayed health-related quality of life and clinical recovery. $\mathrm{J}$ Oral Maxillofac Surg. 2005; 63:55. [PubMed: 15635558]

31. Larrazabal C, Garcia B, Penarrocha M, et al. Influence of oral hygiene and smoking on pain and swelling after surgical extraction of impacted mandibular third molars. J Oral Maxillofac Surg. 2010; 68:43. [PubMed: 20006153] 


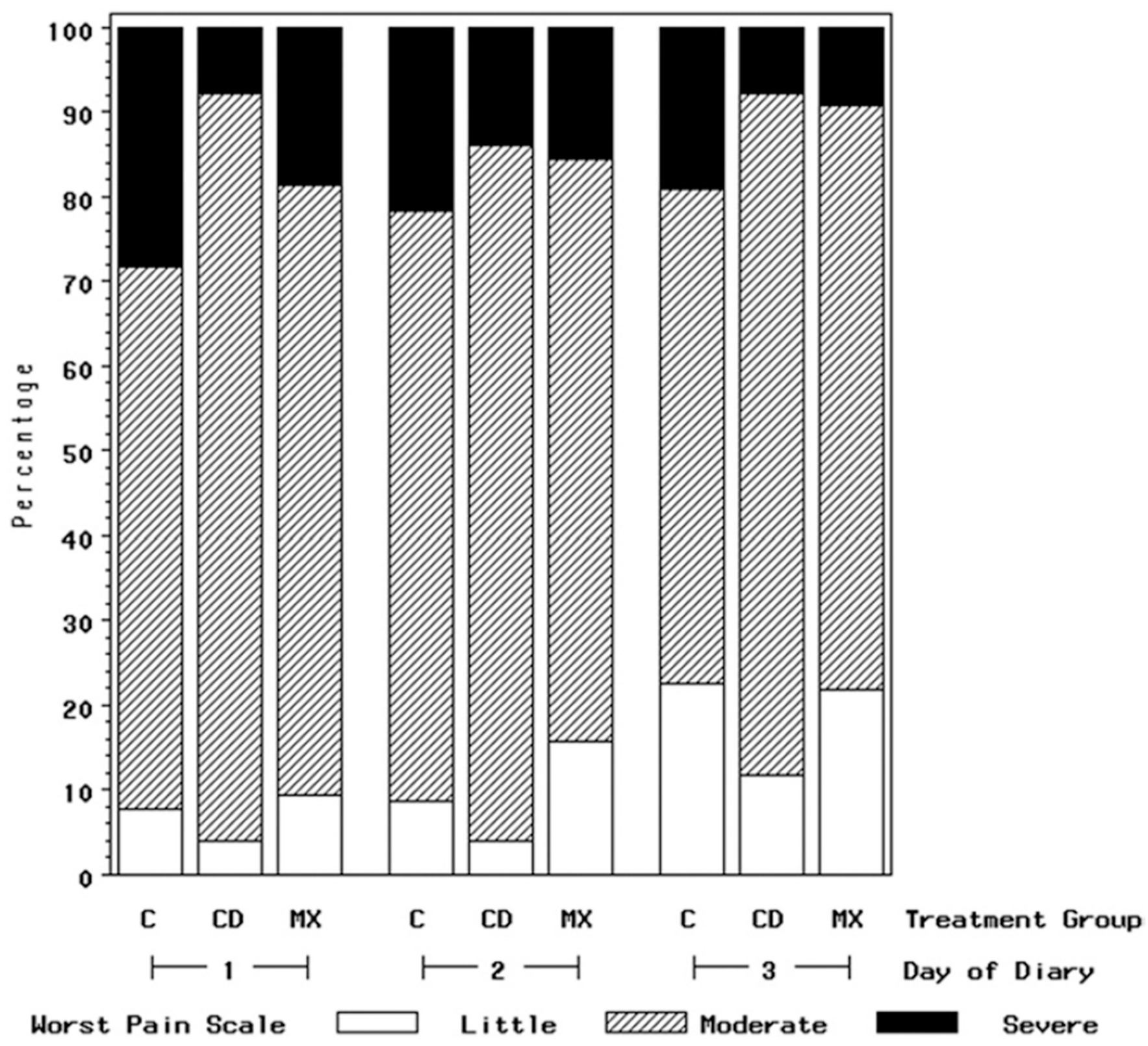

FIGURE 1.

Percent of subjects who reported worst pain on Likert-type scales as little (1-2), moderate (3-5), or severe (6-7) for PSD 1 to 3 for cryotherapy group (CD) $(n=51)$, topical minocycline group (MX) $(n=63)$, and comparison group $(C)(n=92)(N=206)$. It should be noted that the small number of subjects who reported the highest pain scores precluded adequate multivariate statistical analyses.

Gelesko et al. Cryotherapy/Minocycline for Pain Control. J Oral Maxillofac Surg 2011. 


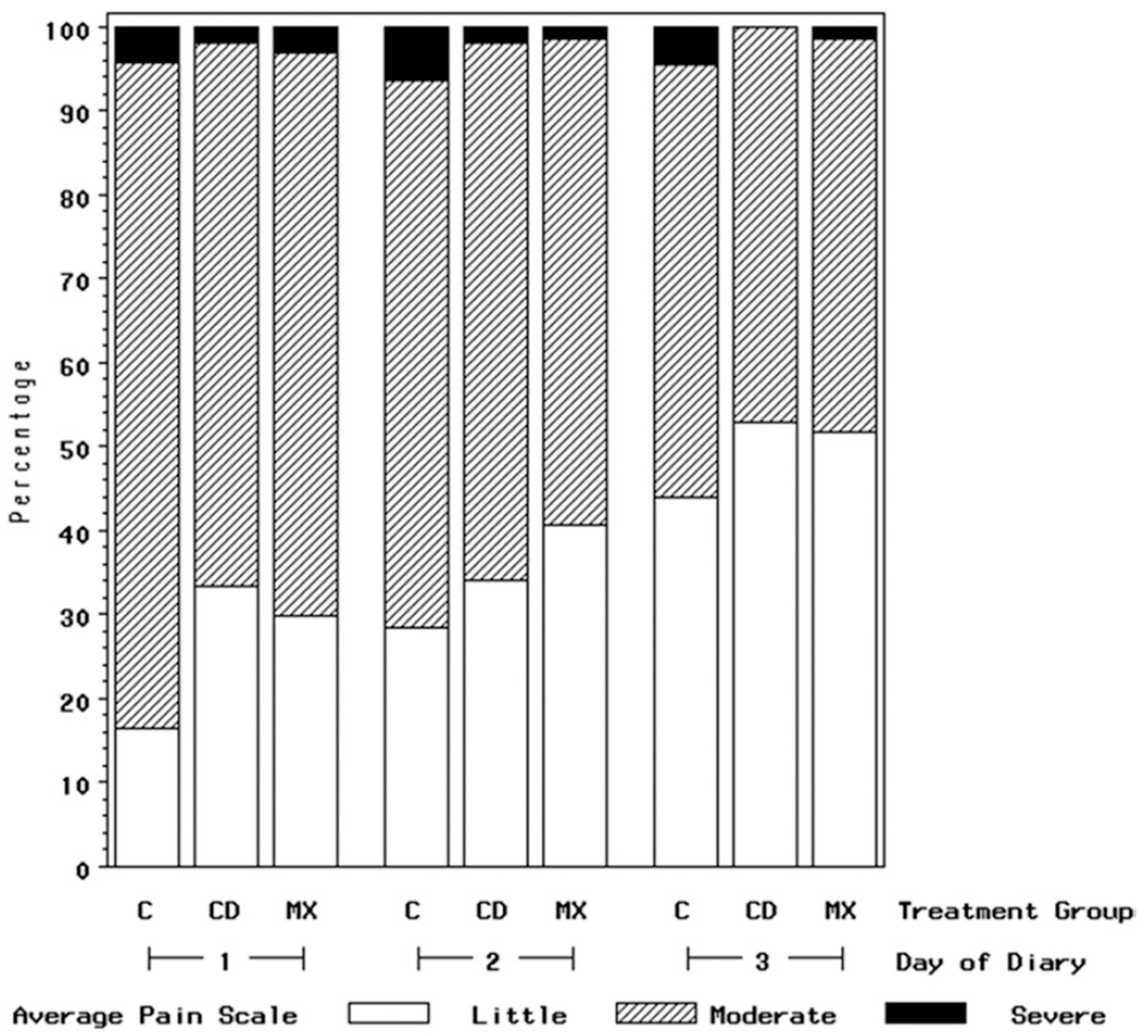

FIGURE 2.

Percent of subjects who reported average pain on Likert-type scales as little (1-2), moderate (3-5), or severe (6-7) for PSD 1 to 3 for cryotherapy group (CD) $(n=51)$, topical minocycline group (MX) $(n=63)$, and comparison group $(C)(n=92)(N=206)$. It should be noted that the small number of subjects who reported the highest pain scores precluded adequate multivariate statistical analyses.

Gelesko et al. Cryotherapy/Minocycline for Pain Control. J Oral Maxillofac Surg 2011. 
Table 1

Characteristics of Cryotherapy Subjects, Topical Minocycline Subjects, and Comparison Subjects $(\mathrm{N}=206)$

\begin{tabular}{|c|c|c|c|c|}
\hline & $\begin{array}{l}\text { Cryotherapy Subjects } \\
(\mathbf{n}=51)\end{array}$ & $\begin{array}{l}\text { Minocycline Subjects } \\
(\mathbf{n}=63)\end{array}$ & $\begin{array}{l}\text { Comparison Subjects } \\
(\mathrm{n}=92)\end{array}$ & $P$ Value \\
\hline Gender $[\mathrm{n}(\%)]$ & & & & .25 \\
\hline M & $27(47)$ & $25(40)$ & $36(39)$ & \\
\hline $\mathrm{F}$ & $24(53)$ & $38(60)$ & $56(61)$ & \\
\hline Race $[\mathrm{n}(\%)]^{*}$ & & & & .70 \\
\hline White & $38(75)$ & $55(87)$ & $71(79)$ & \\
\hline African American & $4(8)$ & $2(3)$ & $10(11)$ & \\
\hline Other & $9(17)$ & $6(10)$ & $9(10)$ & \\
\hline $\begin{array}{l}\text { Bone removal from both mandibular third molars [n } \\
(\%)]^{\dagger}\end{array}$ & $33(65)$ & $42(62)$ & $76(83)$ & .08 \\
\hline Mandibular occlusal plane position [n (\%)] & & & & .04 \\
\hline Third molars at plane & $28(62)$ & $22(37)$ & $39(52)$ & \\
\hline Third molars below plane & $17(38)$ & $37(63)$ & $36(48)$ & \\
\hline Age at surgery [median (IQR)] (yr) & $21(19-24)$ & $21(19-23)$ & $20(18-24)$ & .15 \\
\hline Total surgery time [median (IQR)] (min) & $25(20-40)$ & $27(20-40)$ & $25(18-30)$ & .03 \\
\hline $\begin{array}{l}\text { Surgeon's assessment of degree of difficulty (4-28 } \\
\text { possible) [median (IQR)] }\end{array}$ & $14(11-17)$ & $14(10-18)$ & $12(9-16)$ & .09 \\
\hline
\end{tabular}

NOTE. Cryotherapy subjects were included from 2005 to 2009 from 1 academic center. Topical minocycline subjects were included from 2003 to 2004 from 2 academic centers and 2 community practices. Comparison subjects were included from 2002 to 2006 from 3 academic centers and 2 community practices. Differences between comparison and treatment groups for demographic characteristics and extensiveness of surgery were assessed with $\chi^{2}$ or Cochran-Mantel-Haenszel row mean score tests. The level of significance was set at $P<.05$.

Abbreviation: IQR, interquartile range.

Missing 2 subjects in comparison group.

Missing 5 subjects in minocycline group.

Gelesko et al. Cryotherapy/Minocycline for Pain Control. J Oral Maxillofac Surg 2011. 


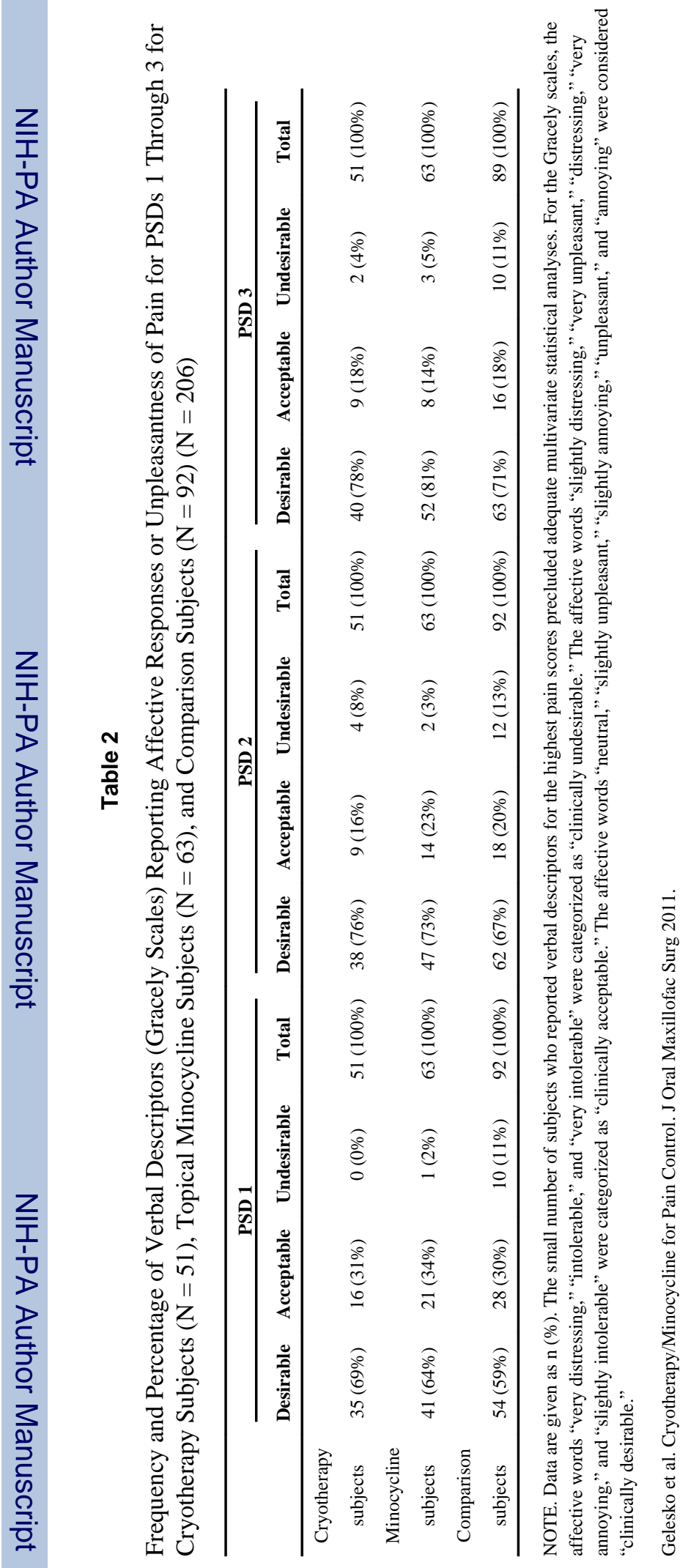




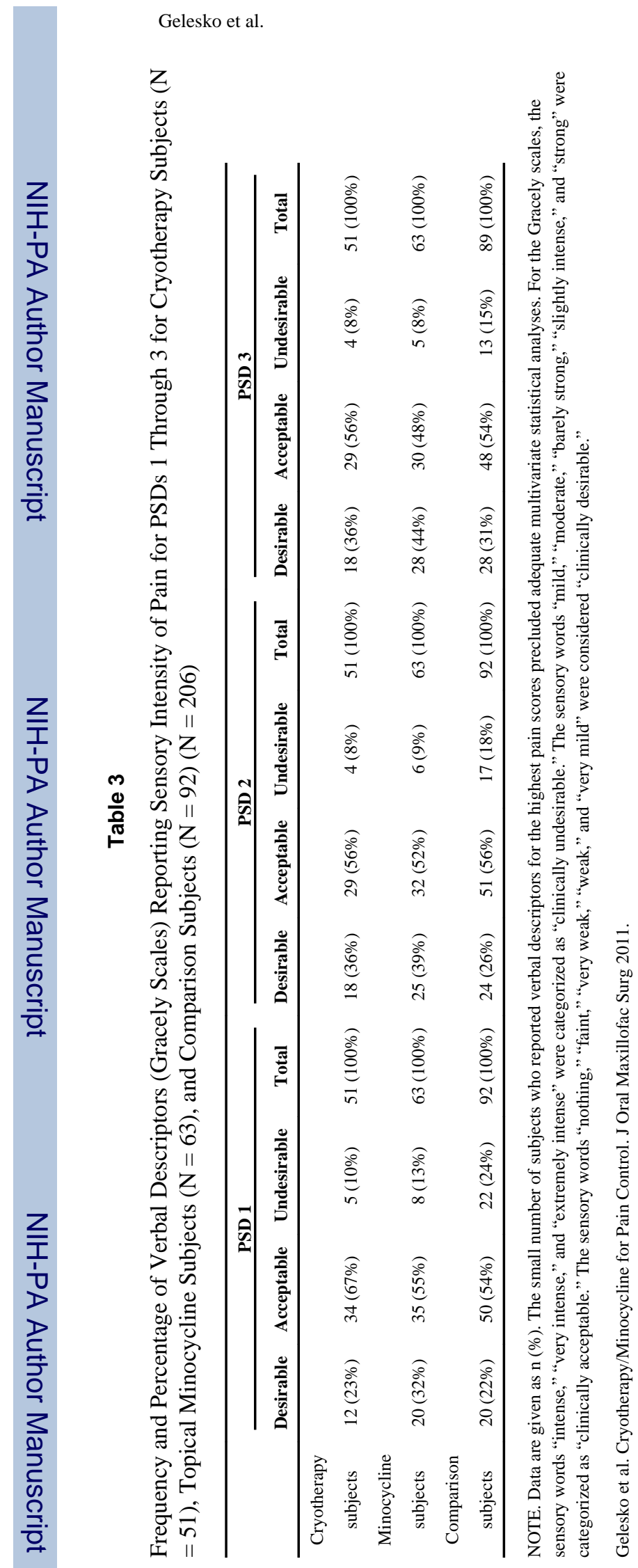

J Oral Maxillofac Surg. Author manuscript; available in PMC 2013 March 05. 